\title{
Primary nonadherence to chronic disease medications: a meta-analysis
}

This article was published in the following Dove Press journal:

Patient Preference and Adherence

\author{
Mark Lemstra' \\ Chijioke Nwankwo ${ }^{2}$ \\ Yelena Bird ${ }^{2}$ \\ John Moraros² \\ 'Alliance Health Medical Clinics, \\ Moose Jaw, Regina and Saskatoon, \\ Saskatchewan, Canada; ${ }^{2}$ School \\ of Public Health, University \\ of Saskatchewan, Saskatoon, \\ Saskatchewan, Canada
}

\begin{abstract}
Background: Medication nonadherence is a global problem that requires urgent attention. Primary nonadherence occurs when a patient consults with a medical doctor, receives a referral for medical therapy but never fills the first dispensation for the prescription medication. Nonadherence to chronic disease medications costs the USA $\sim$ \$290 billion (USD) every year in avoidable health care costs. In Canada, it is estimated that $5.4 \%$ of all hospitalizations are due to medication nonadherence.
\end{abstract}

Objectives: The objective of this study was to quantify the extent of primary nonadherence for four of the most common chronic disease medications. The second objective was to identify factors associated with primary nonadherence to chronic disease medications.

Materials and methods: We conducted an extensive systematic literature review of eight databases with a wide range of keywords. We identified relevant articles for primary nonadherence to antihypertensives, lipid-lowering agents, hypoglycemics, and antidepressants. After further screening and assessment of methodologic quality, relevant data were extracted and analyzed using a random-effects model.

Results: Twenty-four articles were included for our meta-analysis after full review and assessment for risk of bias. The pooled primary nonadherence rate for the four chronic disease medications was $14.6 \%$ (95\% CI: $13.1 \%-16.2 \%)$. Primary medication nonadherence was higher for lipid-lowering medications among the four chronic disease medications assessed $(20.8 \%$; 95\% CI: 16.0\%-25.6\%). The rates in North America (17.0\%; 95\% CI: 14.4\%-19.5\%) were twice those from Europe (8.5\%; 95\% CI: $7.1 \%-9.9 \%)$. The absence of social support $(20 \%$; 95\% CI: $14.4 \%-26.6 \%$ ) was the most common sociodemographic variable associated with chronic disease medication primary nonadherence.

Conclusion: Evidence suggests that a considerable percentage of patients do not initially fill their medications for treatable chronic diseases or conditions. This represents a major health care problem that can be successfully addressed. Efforts should be directed toward proper medication counseling, patient social support, and clinical follow-up, especially when the indications for the prescribed medication aim to provide primary prevention.

Keywords: primary nonadherence, chronic disease medication, initial nonadherence, prescribed medications, predictors of primary nonadherence

\section{Introduction}

The World Health Organization (WHO) reviewed the literature on secondary nonadherence to chronic disease prescription medications and concluded the following: 1) medications do not work if patients do not take them, 2) medication nonadherence is a worldwide problem that crosses all jurisdictions, 3 ) the prevalence of medication nonadherence is of striking magnitude, and 4) this complex issue should be an urgent priority for policy makers and health care providers. ${ }^{1}$ The analysis from the WHO was restricted to secondary nonadherence (patient quits taking medications after starting medical therapy).
Correspondence: Mark Lemstra
Alliance Health Medical Clinics, B70 500 - Ist Avenue NW, Moose Jaw, SK S6H 3M5, Canada Email marklemstra@shaw.ca
Patient Preference and Adherence 2018:12 721-731

721 
Primary nonadherence occurs when a patient consults with a medical doctor, receives a referral for medical therapy, but never fills the first dispensation for the prescription medication. ${ }^{2}$ There are few articles published in the medical literature on primary nonadherence to prescription medications. ${ }^{1}$ For example, in the province of Saskatchewan, Canada, the Health Quality Council concluded that only 29\% of patients fill prescriptions for statin medications within 90 days of being hospitalized for a heart attack. ${ }^{3}$

The impact of medication nonadherence is significant. A research article from Canada demonstrated that 5.4\% of all hospitalizations were due to medication nonadherence and that the subsequent total annual cost burden is as high as $\$ 1.6$ billion Canadian dollars. ${ }^{4}$ Estimates from a study in the USA suggest that nonadherence to chronic disease medications cost the health care system $\$ 290$ billion American dollars every year. ${ }^{5,6}$ Besides the cost implications, the impact to human health and quality of life could be enormous. Medication nonadherence is of paramount concern as current evidence suggests that placing an emphasis on efforts to address this important issue could potentially save more lives than discovering new medical innovations to tackle the conditions for which these medications are prescribed. ${ }^{7,8}$

Global improvements in care and prolonged life expectancy have led not only to an increase in the burden of chronic diseases but also a consequent rise in the number of medication prescriptions and increased budgetary spending on chronic diseases. ${ }^{9}$ Chronic conditions such as hypertension, diabetes, and hyperlipidemia are among the predominant chronic conditions, and these ailments contribute directly and indirectly to $68 \%$ of all deaths worldwide. ${ }^{10}$ Though not commonly categorized as a chronic condition, depression is the most disabling condition worldwide, contributing significantly to disease and medication prescription burden. ${ }^{11}$

Clearly, addressing the issues concerning medication nonadherence can have far-reaching implications toward improving the health and well-being of individuals and entire populations. The amount of research work published on primary medication nonadherence (PMNA) is varied with wideranging estimates of the effect size..$^{3,12-17}$ As such, this study seeks to obtain a pooled estimate of the impact of primary nonadherence to chronic disease medications and identify factors that might be contributing to this important issue.

\section{Materials and methods}

\section{Data sources and study selection}

This study sought to determine the PMNA rate to four common chronic disease medications. The four medication categories considered were antihypertensives, lipid-lowering agents, hypoglycemics, and antidepressants.

PMNA was determined in one of two ways: 1) the proportion of participants who failed to pick up a medication prescribed by their health professional (patient level of measurement) or 2) the proportion of all prescriptions that were not picked up within a specified time (prescription level of measurement). ${ }^{18}$ Measurements made at the patient level can over- or underestimate the true PMNA, which is typically much closer to measurements made at the prescription level. ${ }^{19}$

We conducted an extensive systematic search of the following electronic databases: Cumulative Index of Nursing and Allied Health Literature (CINAHL), Cochrane central, Embase, MEDLINE, ProQuest, PsycINFO, PubMed, and Scopus. Our search was conducted using a combination of several keywords outlined in the search strategy for each database searched (Supplementary materials).

In determining the articles to be included in this study, the authors first eliminated duplicates using the EndNote reference management software. The remaining articles were then screened by their titles and abstracts for relevance. Afterward, two of the authors (CN and ML) reviewed the full-text articles independently for relevance and agreement with the predetermined inclusion criteria (Supplementary materials) to obtain the final articles to be included for the analysis. Methodologic quality and the risk of bias were also independently assessed by two reviewers (CN and ML) by using an adaptation of the Cochrane risk-of-bias tool ${ }^{20}$ and a modified version of the Newcastle-Ottawa scale. ${ }^{21}$ Any disagreements between the reviewing authors (CN and ML) were further discussed and deliberated upon for a possible resolution, and when an agreement was still not possible, a tie-breaking vote was cast by the final author (JM).

\section{Data extraction}

PMNA rates alongside the total number of participants (n) in each study were extracted from each of the included studies. Other relevant information extracted from each study included the type of medication prescribed, the duration of follow-up or observation, the study design, whether the data were from large administrative databases or smaller hospital databases, the average age of study participants, the study location, and the presence or absence of social support (which was defined as some form of routine contact between the health care professional and the participants either through regular follow-ups, text messages, or calls; with the principal aim of improving medication adherence). 


\section{Data analysis}

The $95 \% \mathrm{CI}$ of the included studies was determined using the extracted proportions and the sample size (n). The pooled estimate was obtained using a random-effects model to account for clinical heterogeneity. Heterogeneity was statistically assessed using Higgins I-squared ${ }^{22}$ and further explored with the aid of a subgroup analysis based on categorizations determined a priori. An influence analysis using Tobias' method $^{23}$ was carried out to ascertain the robustness and effect each individual study had on the overall pooled estimate. This involved re-estimating the pooled effect with each study omitted in turn and then assessing whether the overall estimate was skewed significantly. Publication bias was assessed statistically using Begg's test. ${ }^{24}$ All analyses used the "metaprop_one" command and were performed with STATA version 13.1.

\section{Results}

\section{Study selection}

A total of 1,492 articles were obtained from the initial search and this was narrowed to 959 articles after deduplication by use of the reference management software. Of the remaining studies, 894 were found to be unrelated to our study and, therefore, removed after careful screening by their titles and abstracts. Guided by the inclusion and exclusion criteria determined a priori, complete copies of the 65 remaining articles were obtained and further screened for relevance. After reviewing the full articles, 24 studies $^{3,12,14-16,19,25-42}$ were deemed appropriate for inclusion and underwent risk-ofbias assessment and further analysis (Figure 1). A detailed description of the studies included is provided in Table 1.

\section{Risk-of-bias assessment}

Of the selected final 24 articles, 13 were determined to have a low risk of bias, eight were unclear, and three had a high risk of bias. The main methodology concerns among the included experimental studies were centered on performance bias (besides the intervention of interest, researchers acted or treated participants in control or treatment group differently) and detection bias (systematic differences in the measurement of the outcome across both groups). The strengths among the experimental studies included adequate outcome data at follow-up and proper concealment of participant allocation. For the observational studies included for analysis,

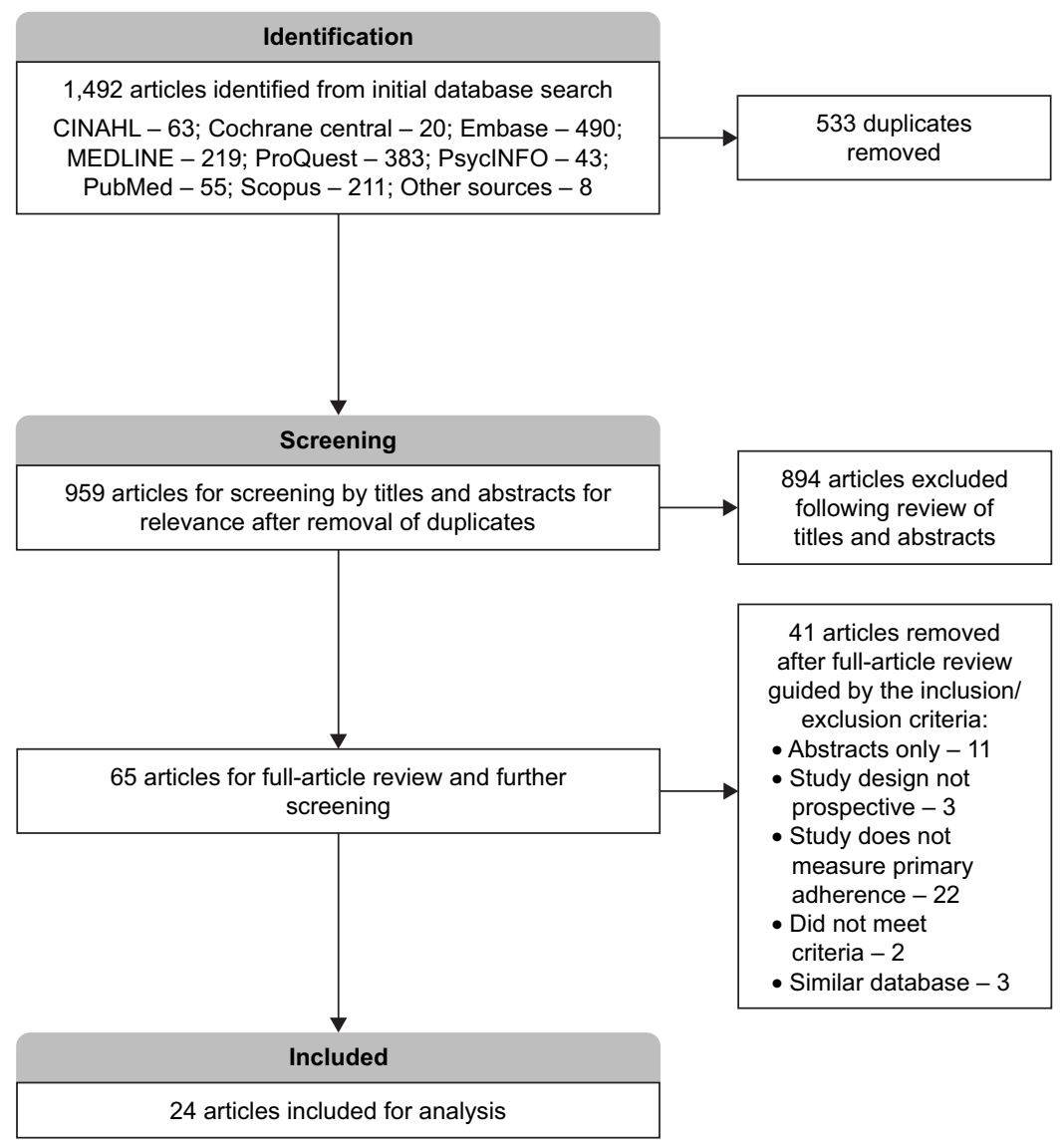

Figure I Prisma flow diagram for included studies. 
Table I Description of included studies

\begin{tabular}{|c|c|c|c|c|c|c|c|}
\hline $\begin{array}{l}\text { Study and } \\
\text { study location }\end{array}$ & $\begin{array}{l}\text { Prescribed } \\
\text { medication class }\end{array}$ & $\begin{array}{l}\text { Duration of } \\
\text { follow-up }\end{array}$ & $\begin{array}{l}\text { Study } \\
\text { design }\end{array}$ & $\begin{array}{l}\text { Average/ } \\
\text { median age } \\
\text { (years) }\end{array}$ & Database & $\begin{array}{l}\text { Level of } \\
\text { measurement }\end{array}$ & $\begin{array}{l}\text { Predictors of primary } \\
\text { nonadherence }\end{array}$ \\
\hline $\begin{array}{l}\text { Aznar-Lou et al;,26 } \\
2017 \text {, Spain }\end{array}$ & $\begin{array}{l}\text { Hypoglycemics } \\
\text { Antihypertensives } \\
\text { Lipid-lowering } \\
\text { Antidepressants }\end{array}$ & I month & R.cohort & 52.4 & Administrative & Prescription & $\begin{array}{l}\text { No comorbidities, diseases } \\
\text { other than diabetes, young } \\
\text { female prescribing GP, GP } \\
\text { in training }\end{array}$ \\
\hline $\begin{array}{l}\text { Bauer et al:;5 } \\
2014 \text {, USA }\end{array}$ & Antidepressants & 2 months & R.cohort & 58 & Administrative & Patient & $\begin{array}{l}\text { Lack of involvement } \\
\text { in decision making }\end{array}$ \\
\hline $\begin{array}{l}\text { Casebeer et al; }{ }^{27} \\
2009, \text { USA }\end{array}$ & Lipid-lowering & 4 months & CT & 58 & Hospital & Patient & $\begin{array}{l}\text { No social support: } \\
\text { absence of educational } \\
\text { programs }\end{array}$ \\
\hline $\begin{array}{l}\text { Chan et al; }{ }^{3} \\
\text { 2004, Canada }\end{array}$ & $\begin{array}{l}\text { Antihypertensives } \\
\text { Lipid-lowering }\end{array}$ & 60 months & R.cohort & 70 & Hospital & Patient & - \\
\hline $\begin{array}{l}\text { Cheetham et al; }{ }^{28} \\
2013, \text { USA }\end{array}$ & Lipid-lowering & 3 months & R.cohort & $57^{\mathrm{a}}$ & Administrative & Patient & Black race, polymedication \\
\hline $\begin{array}{l}\text { Derose et al; }{ }^{29} \\
2013, \text { USA }\end{array}$ & Lipid-lowering & 3 months & RCT & 56.1 & Administrative & Patient & $\begin{array}{l}\text { No social support: } \\
\text { absence of text reminder, } \\
\text { no drug coverage }\end{array}$ \\
\hline $\begin{array}{l}\text { Ewen et al; }{ }^{30} \\
2015 \text {, Germany }\end{array}$ & Antihypertensives & 6 months & P.cohort & 62.7 & Hospital & Patient & - \\
\hline $\begin{array}{l}\text { Fischer et al; }{ }^{31} \\
2015, \text { USA }\end{array}$ & $\begin{array}{l}\text { Hypoglycemics } \\
\text { Antihypertensives } \\
\text { Lipid-lowering }\end{array}$ & 0.5 months & $\mathrm{RCT}$ & 53.2 & Hospital & Patient & - \\
\hline $\begin{array}{l}\text { Fischer et al;12 } \\
2010, \text { USA }\end{array}$ & $\begin{array}{l}\text { Hypoglycemics } \\
\text { Antihypertensives } \\
\text { Lipid-lowering } \\
\text { Antidepressants }\end{array}$ & 12 months & R.cohort & 44.3 & Administrative & Prescription & New prescriptions \\
\hline $\begin{array}{l}\text { Freccero et al; }{ }^{32} \\
2016, \text { Sweden }\end{array}$ & Antidepressants & I month & R.cohort & 48.2 & Administrative & Patient & $\begin{array}{l}\text { Country of origin, } \\
\text { young age, marital status } \\
\text { (divorce) }\end{array}$ \\
\hline $\begin{array}{l}\text { van Geffen } \\
\text { et al; }{ }^{33} 2009 \text {, the } \\
\text { Netherland }\end{array}$ & Antidepressants & I month & R.cohort & 48.5 & Hospital & Patient & New prescriptions \\
\hline 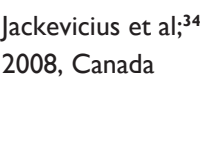 & $\begin{array}{l}\text { Hypoglycemics } \\
\text { Antihypertensives } \\
\text { Lipid-lowering } \\
\text { Antidepressants }\end{array}$ & I month & R.cohort & 76.3 & Administrative & Prescription & $\begin{array}{l}\text { Older age, higher income, } \\
\text { more medications }\end{array}$ \\
\hline $\begin{array}{l}\text { Karter et al;35 } \\
2009, \text { USA }\end{array}$ & $\begin{array}{l}\text { Hypoglycemics } \\
\text { Antihypertensives } \\
\text { Lipid-lowering }\end{array}$ & 2 months & R.cohort & 61.2 & Administrative & Patient & - \\
\hline $\begin{array}{l}\text { Kerner et al;36 } \\
2017, \text { USA }\end{array}$ & Antihypertensives & I month & P.cohort & 63.9 & Hospital & Patient & $\begin{array}{l}\text { No social support: } \\
\text { absence of messages } \\
\text { and calls }\end{array}$ \\
\hline $\begin{array}{l}\text { Linnet et al; } 19 \\
2012 \text {, Iceland }\end{array}$ & $\begin{array}{l}\text { Hypoglycemics } \\
\text { Antihypertensives } \\
\text { Antidepressants }\end{array}$ & I month & R.cohort & - & Administrative & Prescription & Cost \\
\hline $\begin{array}{l}\text { O'Connor et al; }{ }^{37} \\
2014, \text { USA }\end{array}$ & Hypoglycemics & 2 months & RCT & 61.7 & Administrative & Patient & $\begin{array}{l}\text { No social support: } \\
\text { absence of telephone } \\
\text { support }\end{array}$ \\
\hline $\begin{array}{l}\text { Raebel et al;:25 } \\
2012, \text { USA }\end{array}$ & $\begin{array}{l}\text { Hypoglycemics } \\
\text { Antihypertensives } \\
\text { Lipid-lowering }\end{array}$ & I month & R.cohort & 59.2 & Administrative & Patient & $\begin{array}{l}\text { Race, smoking, less care } \\
\text { contacts, comorbidities, } \\
\text { cost }\end{array}$ \\
\hline $\begin{array}{l}\text { Shah et al; }{ }^{38} \\
2008, \text { USA }\end{array}$ & Hypoglycemics & I month & R.cohort & 49 & Administrative & Patient & Cost, good health \\
\hline $\begin{array}{l}\text { Shah et al; }{ }^{39} \\
2009, \text { USA }\end{array}$ & Antihypertensives & I month & R.cohort & 47 & Administrative & Patient & $\begin{array}{l}\text { Female, comorbidities, } \\
\text { older age, less severe } \\
\text { disease }\end{array}$ \\
\hline
\end{tabular}


Table I (Continued)

\begin{tabular}{|c|c|c|c|c|c|c|c|}
\hline $\begin{array}{l}\text { Study and } \\
\text { study location }\end{array}$ & $\begin{array}{l}\text { Prescribed } \\
\text { medication class }\end{array}$ & $\begin{array}{l}\text { Duration of } \\
\text { follow-up }\end{array}$ & $\begin{array}{l}\text { Study } \\
\text { design }\end{array}$ & $\begin{array}{l}\text { Average/ } \\
\text { median age } \\
\text { (years) }\end{array}$ & Database & $\begin{array}{l}\text { Level of } \\
\text { measurement }\end{array}$ & $\begin{array}{l}\text { Predictors of primary } \\
\text { nonadherence }\end{array}$ \\
\hline $\begin{array}{l}\text { Shin et al;'16 } \\
2012 \text {, USA }\end{array}$ & $\begin{array}{l}\text { Hypoglycemics } \\
\text { Antihypertensives } \\
\text { Lipid-lowering } \\
\text { Antidepressants }\end{array}$ & 3 months & R.cohort & 46.5 & Administrative & Prescription & $\begin{array}{l}\text { Minority race, lower } \\
\text { income, greater number } \\
\text { of prescriptions on the } \\
\text { index date }\end{array}$ \\
\hline $\begin{array}{l}\text { Tamblyn et al; }{ }^{40} \\
2014, \text { Canada }\end{array}$ & $\begin{array}{l}\text { Hypoglycemics } \\
\text { Antihypertensives } \\
\text { Lipid-lowering }\end{array}$ & 9 months & P.cohort & 61.6 & Hospital & Prescription & $\begin{array}{l}\text { New prescriptions, } \\
\text { young age, cost, lower } \\
\text { health visits }\end{array}$ \\
\hline $\begin{array}{l}\text { Thengilsdóttir } \\
\text { et al; }{ }^{14} 2015, \\
\text { Iceland }\end{array}$ & $\begin{array}{l}\text { Lipid-lowering } \\
\text { Antidepressants }\end{array}$ & 12 months & R.cohort & $\begin{array}{l}58.7 \\
45.4\end{array}$ & Administrative & Prescription & Female gender, cost \\
\hline $\begin{array}{l}\text { Trinacty et al; } \\
2009, \text { USA }\end{array}$ & Hypoglycemics & I month & R.cohort & 51 & Administrative & Patient & - \\
\hline $\begin{array}{l}\text { Xing et al; }{ }^{42} \\
2011 \text {, USA }\end{array}$ & Antidepressants & 24 months & R.cohort & 51.5 & Administrative & Prescription & $\begin{array}{l}\text { New prescriptions, } \\
\text { young age }\end{array}$ \\
\hline
\end{tabular}

Notes: Database: the data source, that is, administrative (from large admin databases), hospital (from clinic or hospital records). Level of measurement: primary nonadherence could have been measured as the proportion of participants (patient level of measurement) failing to fill their prescription or the proportion of prescriptions not filled

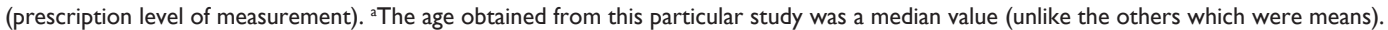

Abbreviations: CT, controlled trial; GP, General Practitioner; PMNA, primary medication nonadherence; P.cohort, prospective cohort; R.cohort, retrospective cohort; $\mathrm{RCT}$, randomized controlled trial.

selection of the cohort of interest was adequate and there was minimal bias noted with the comparability of the cohorts and assessment of the outcome (Figure 2A and B).

\section{Characteristics of the pool}

A total of 550,485 prescriptions were pooled from the 24 included studies, with 64,892 of those prescriptions not being redeemed within the defined period (Table 2). Seventeen of the included studies assessed PMNA by following up 467,483 prescriptions for a 3-month duration or less, ${ }^{15,16,19,25,26,28,29,31-39,41}$ while seven studies assessed PMNA among 83,002 prescriptions over an extended time-frame (ie, >3 months). ${ }^{3,12,14,27,30,40,42}$ Eight studies determined PMNA at the level of the prescription. ${ }^{12,14,16,19,26,34,40,42}$ The highest number of chronic disease medication prescriptions identified in this study were for antihypertensives $(190,658)$, followed closely by antidepressants $(164,542)$, lipid-lowering medications $(149,714)$, and hypoglycemics $(45,571)$. A majority (16) of the studies were retrospective cohort studies, ${ }^{3,12,14-16,}$ $19,25,26,28,32-35,38,39,41,42$ while seven studies were either prospective cohorts or clinical trials. ${ }^{27,29-31,36,37,40}$ Six of the included studies were conducted in Europe, ${ }^{14,19,26,30,32,33}$ while the rest were in either the United States or Canada. ${ }^{3,12,15,16,25,27-29,31,34-36,37-42}$ All but seven of the studies utilized data from large administrative databases $^{9,14-16,19,25,26,28,29,32,34,35,37-39,41,42}$ (Table 3).

\section{Pooled analyses}

Overall, the pooled estimates showed that the incidence of PMNA for the four most common chronic diseases was
14.6\% (95\% CI: 13.1\%-16.2\%) (Table 1; Figure 3). These estimates were unlikely to be influenced by bias, as PMNA did not differ significantly in the studies with a low risk of bias when compared to those with an unclear or high risk of bias (Table 3). Variation between the studies was addressed using a random-effects model and a subgroup analysis was carried out to further explore the potential reasons for between-study variations.

The following findings were of interest. The only sociodemographic variable with a consistent association with PMNA was lack of social support. Those without social support had higher rates of PMNA (20\%; 95\% CI: 14.4\%-26.6\%) than those with social support (13.1\%; 95\% CI: 11.4\%-14.8\%). Other variables, like age, demonstrated inconsistent associations. PMNA for lipid-lowering medications like statins (20.8\%; 95\% CI: 16.0\%-25.6\%) was higher than the PMNA rates for antihypertensives $(12.4 \%$; 95\% CI: 9.5\%-15.3\%), hypoglycemics $(13.2 \%$; 95\% CI: 9.6\%-16.8\%), and antidepressants (10.8\%; 95\% CI: 8.2\%-13.4\%). The extent of PMNA for North America (17.0\%; 95\% CI: 14.4\%-19.5\%) was twice the rate estimated for Europe $(8.5 \%$; 95\% CI: $7.1 \%-9.9 \%$ ). Another significant finding was that studies with prescription follow-up lasting $>3$ months $(25.3 \%$; 95\% CI: $19.7 \%-30.9 \%$ ) had more than twice the PMNA, compared to those where the studies follow patients for 3 months or less $(10.0 \%$; 95\% CI: $8.7 \%-11.4 \%)$. Where prospective cohort study designs or clinical trials were used, PMNA was higher, but not significant, compared to 


\section{A}

Random sequence generation

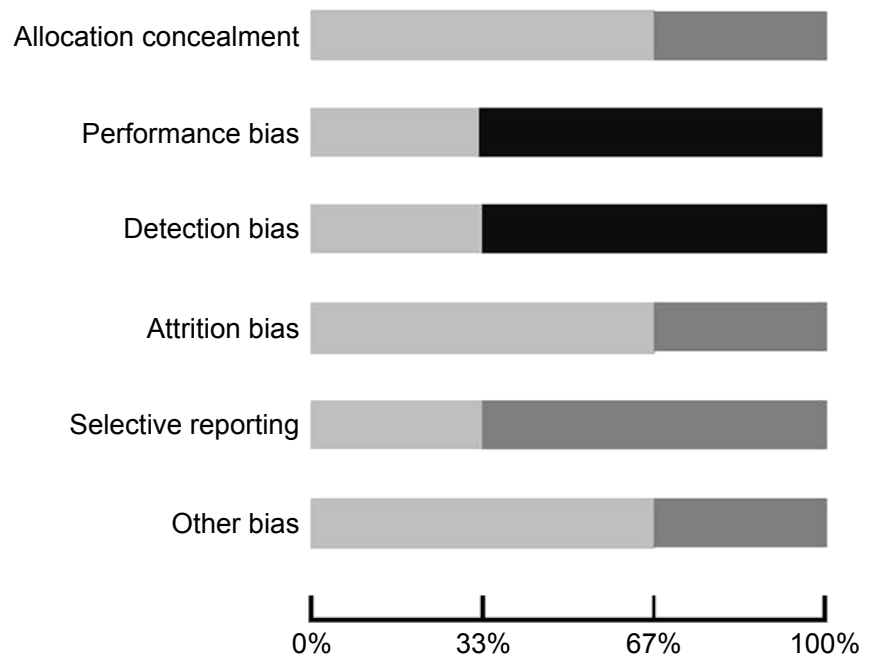

B

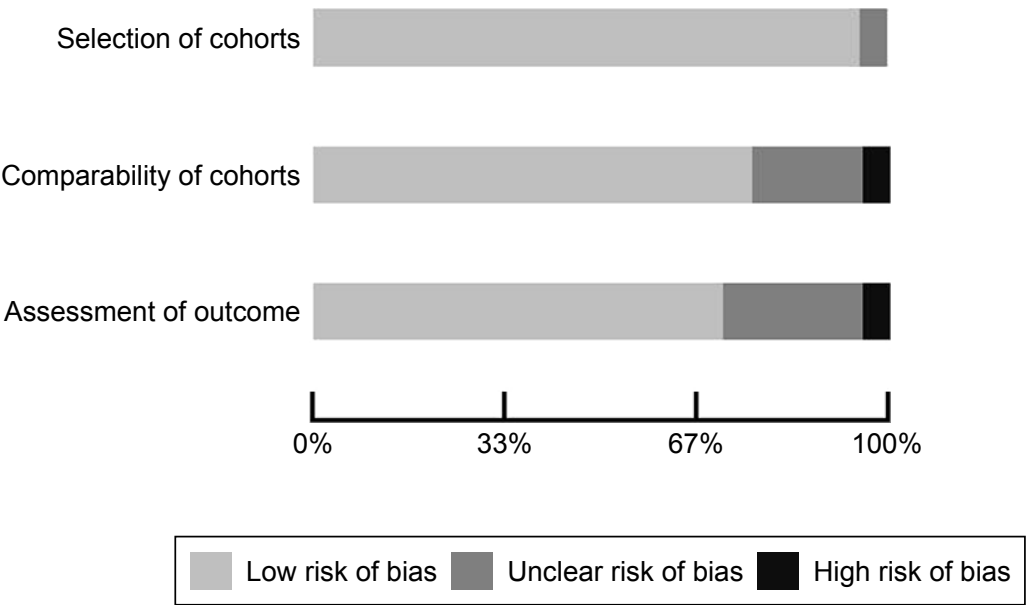

Figure 2 (A) Risk-of-bias plot - experimental studies. (B) Risk-of-bias plot - observational studies.

retrospective cohort studies. Similarly, PMNA was higher for studies obtained from hospital databases compared to those from large administrative databases but the association was not significant (Table 3 ).

Influential analyses carried out following Tobias' method showed that the pooled estimates did not vary significantly with the exclusion of any one study. This suggests that none of the studies had a significant influential effect on the

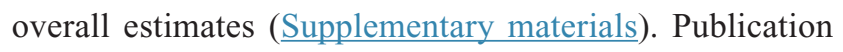
bias was assessed statistically using Begg's test. The test was not statistically significant (adjusted Kendall's score $=208$, $P=0.066$ ), suggesting that publication bias was unlikely.

\section{Discussion}

The WHO has identified the issue of medication nonadherence as a global concern and one that requires urgent intervention. ${ }^{1}$
In our meta-analysis, we found that on average 15 of every 100 medications prescribed for chronic diseases or conditions are not initially filled by patients. Cost barriers play a key role in promoting medication non-adherence. ${ }^{43}$

For secondary nonadherence to chronic disease medications, two meta-analyses reviewed nonadherence to statins (49.0\%; 95\% CI: 48.9\%-49.2\%) and antihypertensive medications (48.5\%; 95\% CI: 47.7\%-49.2\%) in real-world settings. ${ }^{2,44}$ If we put together the findings from these two meta-analyses on secondary nonadherence, along with the findings from our meta-analysis that showed primary nonadherence of $14.6 \%$, we can extrapolate that $\sim 41.8 \%$ of patients are adherent to chronic disease or chronic condition medications ( $49 \%$ of $85.4 \%$ equals $41.8 \%$ ). Placing these figures alongside the cost estimates from the New England Health Institute, one can preliminarily ascertain that if steps 
Table 2 Pooled estimates

\begin{tabular}{|c|c|c|c|c|}
\hline $\begin{array}{l}\text { Study and study } \\
\text { location }\end{array}$ & $\begin{array}{l}\text { Prescribed } \\
\text { medication } \\
\text { class }\end{array}$ & $\begin{array}{l}\text { Number } \\
\text { of study } \\
\text { prescriptions }\end{array}$ & $\begin{array}{l}\text { Primary } \\
\text { nonadherence } \\
\text { rate (\%) }\end{array}$ & $95 \% \mathrm{Cl}$ \\
\hline Aznar-Lou et al; ${ }^{26}$ & Hypoglycemics & 8,270 & 13.2 & $12.5-14.0$ \\
\hline \multirow[t]{3}{*}{ 2017, Spain } & Antihypertensives & 74,346 & 7.5 & 7.3-7.7 \\
\hline & Lipid-lowering & 69,602 & 8.8 & $8.6-9.0$ \\
\hline & Antidepressants & 97,635 & 11.5 & $11.3-11.7$ \\
\hline Bauer et al; $;^{15} 2014$, USA & Antidepressants & 1,523 & 4.3 & $3.3-5.4$ \\
\hline Casebeer et al; ${ }^{27} 2009$, USA & Lipid-lowering & 913 & 43.2 & $39.9-46.4$ \\
\hline \multirow[t]{2}{*}{ Chan et al; ${ }^{3} 2004$, Canada } & Antihypertensives & $\mathrm{I}, 700$ & 33.5 & $31.3-35.8$ \\
\hline & Lipid-lowering & $\mathrm{I}, 700$ & 71.0 & $68.8-73.1$ \\
\hline Cheetham et al: ${ }^{28}$ & Lipid-lowering & 19,826 & 15.4 & $14.9-15.9$ \\
\hline \multicolumn{5}{|l|}{2013, USA } \\
\hline Derose et al:;9 2013 , USA & Lipid-lowering & 5,216 & 18.4 & $17.4-19.5$ \\
\hline Ewen et al; $; 02015$ & Antihypertensives & 100 & 2.0 & $0.2-7.0$ \\
\hline \multicolumn{5}{|l|}{ Germany } \\
\hline \multirow[t]{3}{*}{ Fischer et al; ${ }^{31} 2015$, USA } & Hypoglycemics & 346 & 6.4 & $4.0-9.5$ \\
\hline & Antihypertensives & 2,065 & 3.3 & $2.6-4.2$ \\
\hline & Lipid-lowering & 528 & 6.4 & $4.5-8.9$ \\
\hline \multirow[t]{4}{*}{ Fischer et al; ${ }^{12} 2010$, USA } & Hypoglycemics & 5,525 & 21.9 & $20.8-23.0$ \\
\hline & Antihypertensives & 30,211 & 19.5 & $19.0-19.9$ \\
\hline & Lipid-lowering & 12,963 & 19.9 & $19.2-20.6$ \\
\hline & Antidepressants & 11,767 & 21.4 & $20.6-22.1$ \\
\hline Freccero et al; ${ }^{32}$ & Antidepressants & 11,624 & 14.9 & $14.3-15.6$ \\
\hline \multicolumn{5}{|l|}{ 2016, Sweden } \\
\hline van Geffen et al; ${ }^{33}$ & Antidepressants & 965 & 4.3 & $3.1-5.7$ \\
\hline \multicolumn{5}{|l|}{ 2009, the Netherland } \\
\hline Jackevicius et al; ${ }^{34}$ & Hypoglycemics & 146 & 13.7 & $8.6-20.4$ \\
\hline \multirow[t]{3}{*}{ 2008, Canada } & Antihypertensives & 5,337 & 6.4 & $5.8-7.1$ \\
\hline & Lipid-lowering & 758 & 5.2 & $3.7-7.0$ \\
\hline & Antidepressants & 43 & 32.6 & $19.1-48.5$ \\
\hline \multirow[t]{3}{*}{ Karter et al; ${ }^{35} 2009$, USA } & Hypoglycemics & 8,191 & 4.0 & $3.6-4.5$ \\
\hline & Antihypertensives & $12,7 \mid 2$ & 3.2 & $2.9-3.5$ \\
\hline & Lipid-lowering & 6,426 & 8.5 & $7.8-9.2$ \\
\hline Kerner et al; $;^{36} 2017$, USA & Antihypertensives & 9 & 22.2 & $2.8-60.0$ \\
\hline \multirow[t]{3}{*}{ Linnet et al; ${ }^{19} 2012$, Iceland } & Hypoglycemics & 760 & 8.7 & $6.8-10.9$ \\
\hline & Antihypertensives & 4,127 & 8.6 & $7.7-9.5$ \\
\hline & Antidepressants & 4,492 & 6.6 & $5.9-7.4$ \\
\hline O'Connor et al $; ;^{37} 2014$, USA & Hypoglycemics & 2,378 & 13.3 & $11.9-14.7$ \\
\hline \multirow[t]{3}{*}{ Raebel et al;:25 2012, USA } & Hypoglycemics & $|, 52|$ & 11.3 & $9.8-13.0$ \\
\hline & Antihypertensives & 4,721 & 7.0 & $6.3-7.8$ \\
\hline & Lipid-lowering & 4,607 & 12.6 & $11.6-13.6$ \\
\hline Shah et al: ${ }^{38} 2008$, USA & Hypoglycemics & 1,132 & 15 & $13.0-17.2$ \\
\hline Shah et al:39 2009 , USA & Antihypertensives & 3,240 & 17.1 & $15.8-18.5$ \\
\hline \multirow[t]{4}{*}{ Shin et al; ${ }^{16} 2012$, USA } & Hypoglycemics & 14,417 & 12.6 & $12.0-13.1$ \\
\hline & Antihypertensives & 48,982 & 7.8 & $7.5-8.0$ \\
\hline & Lipid-lowering & 22,249 & 22.3 & $21.8-22.9$ \\
\hline & Antidepressants & 27,383 & 7.7 & $7.4-8.0$ \\
\hline Tamblyn et al; ${ }^{40}$ & Hypoglycemics & 979 & 29.1 & $26.3-32.1$ \\
\hline \multirow[t]{2}{*}{ 2014, Canada } & Antihypertensives & 3,108 & 32.2 & $30.5-33.8$ \\
\hline & Lipid-lowering & 2,794 & 33.6 & $31.9-35.4$ \\
\hline Thengilsdóttir et al; ${ }^{\prime 4}$ & Lipid-lowering & 2,132 & 6.2 & $5.2-7.3$ \\
\hline 2015, Iceland & Antidepressants & 8,553 & 8.0 & $7.4-8.6$ \\
\hline Trinacty et al;"' 2009, USA & Hypoglycemics & 1,906 & 10.0 & $8.7-11.5$ \\
\hline Xing et al $;{ }^{42} 20 \mathrm{I}$, USA & Antidepressants & 557 & 13.1 & $10.4-16.2$ \\
\hline Pooled random estimate & & 550,485 & 14.6 & $13.1-16.2$ \\
\hline
\end{tabular}


Table 3 Subgroup analysis

\begin{tabular}{|c|c|c|}
\hline Subgroup & PMNA $(95 \% \mathrm{Cl})$ & $\mathbf{N}$ \\
\hline \multicolumn{3}{|l|}{ Medication } \\
\hline Hypoglycemics & $13.2(9.6-16.8)$ & 45,571 \\
\hline Antihypertensives & $12.4(9.5-15.3)$ & 190,658 \\
\hline Lipid-lowering & $20.8(16.0-25.6)$ & $|49,7| 4$ \\
\hline Antidepressants & $10.8(8.2-13.4)$ & 164,542 \\
\hline \multicolumn{3}{|l|}{ Duration of follow-up } \\
\hline$\leq 3$ months & $10.0(8.7-11.4)$ & 467,483 \\
\hline$>3$ months & $25.3(19.7-30.9)$ & 83,002 \\
\hline \multicolumn{3}{|l|}{ Study design } \\
\hline R.cohort & | $3.5(|| .8-\mid 5.2)$ & 532,049 \\
\hline P.cohort, CT, RCT & $18.9(\mid 1.0-26.8)$ & 18,436 \\
\hline \multicolumn{3}{|l|}{ Average age, years } \\
\hline 50 or less & |4.8 (II.4-|8.2) & $|99,0| \mid$ \\
\hline $51-60$ & $11.4(9.8-12.9)$ & 295,714 \\
\hline$>60$ & $20.4(\mid 4.9-25.8)$ & 46,381 \\
\hline \multicolumn{3}{|l|}{ Data source } \\
\hline Administrative database & $11.7(10.2-13.3)$ & 535,278 \\
\hline Hospital database & $24.0(12.0-35.9)$ & 15,207 \\
\hline \multicolumn{3}{|l|}{ Level of measurement } \\
\hline Prescription & $14.5(\mid 2.7-16.4)$ & 457,136 \\
\hline Patient & |4.8 (II.4-|8.2) & 93,349 \\
\hline \multicolumn{3}{|l|}{ Risk of bias } \\
\hline Low risk & $12.9(\mid 1.2-14.5)$ & 493,728 \\
\hline Unclear/high risk & $17.3(|3.0-2| .5)$ & 56,757 \\
\hline \multicolumn{3}{|l|}{ Location } \\
\hline North America & $17.0(\mid 4.4-19.5)$ & 267,879 \\
\hline Europe & $8.5(7.1-9.9)$ & 282,606 \\
\hline \multicolumn{3}{|l|}{ Absence of social support } \\
\hline Yes & $20.5(14.4-26.6)$ & 27,769 \\
\hline No & |3.| (| | $.4-\mid 4.8)$ & 522,716 \\
\hline
\end{tabular}

Abbreviations: $C T$, controlled trial; $N$, total number of prescriptions; PMNA, primary medication nonadherence; P.cohort, prospective cohort; RCT, randomized controlled trial; R.cohort, retrospective cohort.

were taken to reduce the PMNA rate for chronic disease medications by even $1 \%$ (on an absolute level - not relative) it can potentially save the US health care system $\sim \$ 2.9$ billion (USD) annually. ${ }^{6}$

Our subgroup analysis showed that lipid-lowering medications like statins had the highest rate of PMNA for the chronic disease medications (20.8\%). A plausible explanation for the observed high PMNA rate is that these medications are often used for primary prevention and, therefore, patients may feel that there is no immediate threat to their health. ${ }^{2,45}$ This was not the case with hypoglycemic or antidepressant medications, where the common indications for use have clear morbidity and mortality implications that can be easily recognized by patients.

Additionally, we found a difference between the PMNA rates for chronic disease medications when we stratified by the location of the study. Prescriptions for medications based out of Europe had a PMNA rate of $8.5 \%$, while those from North America had PMNA rates of $17.0 \%$. These differences may be related to the variations in the delivery of care and the cost of health care in these regions. In most cases, European nations have stronger social programs that include universal health care coverage with a greater percentage of public funding. ${ }^{46,47}$ On the other hand, the North American studies were predominantly from the USA, where universal coverage is limited and there is greater dependence on private insurance systems. ${ }^{47}$

Our subgroup analysis by the duration of follow-up showed that PMNA rates for studies with a longer period of follow-up (3 months or more) were more than double the rates for those with shorter follow-up (3 months or less). This is expected, as the longer the study duration, the more likely nonadherence will be detected. ${ }^{27}$ For example, in one study, it took patients an average of $\sim 2$ years to fill their first prescription for statin medications. $^{2}$

The absence of social support was also noted to play a key role in negatively impacting the PMNA rates. These findings are not surprising, as studies in the past have shown a clear relationship between the absence of social support and patient nonadherence. ${ }^{15,27,29,36,37,48,49}$ Given that social support from clinicians, family members, and friends is a modifiable factor, this variable represents an area of interest and further research.

The Cochrane Collaboration reviewed the literature on the impact of social support on medication nonadherence and concluded that more frequent interaction between doctors and patients was the most effective intervention. ${ }^{50}$ A second meta-analysis from the Cochrane Collaboration reviewed interventions to specifically improve adherence to lipidlowering medications. Overall, only one of four patients continued to take medications in the long term. In this review, patient reinforcement and regular reminders were the most promising interventions. The authors concluded that a combination of strategies including reminders, reinforcement, and emphasis on appreciating the patient's perspective might lead to the most effective strategy. ${ }^{51}$ Similarly, the National Collaborating Centre for Primary Care performed a systematic review of the literature and advocated that health care professionals 1) adapt their consultation style to the needs of individual patients, 2) make information more accessible and easy to understand for their patients, 3) increase patient involvement in decision making, 4) be aware that patients may have concerns about their prescribed medicines, and 5) recognize that nonadherence is quite common and that they should routinely assess for it in a nonjudgmental way. ${ }^{52}$

In summary, our meta-analysis helps to provide a more clear and accurate picture of the burden of PMNA, while identifying a number of associated factors. Health care professionals and policy makers should place more emphasis 


\section{Study}

Aznar-Lou et al;26 2017 (a)

Aznar-Lou et al; ${ }^{26} 2017$ (b)

Aznar-Lou et al; ${ }^{26} 2017$ (c)

Aznar-Lou et al;:26 2017 (d)

Bauer et al; ;15 2014

Casebeer et al; ${ }^{27} 2009$

Chan et al; 2004 (b)

Chan et al; 2004 (c)

Cheetham et al:;8 2013

Derose et al; ${ }^{29} 2013$

Ewen et al; ${ }^{30} 2015$

Fischer et al; ${ }^{31} 2015$ (a)

Fischer et al; ${ }^{31} 2015$ (b)

Fischer et al; ${ }^{31} 2015$ (c)

Fischer et al; ${ }^{12} 2010$ (a)

Fischer et al; ${ }^{12} 2010$ (b)

Fischer et al;:12 2010 (c)

Fischer et al;:12 2010 (d)

Freccero et al; ${ }^{32} 2016$

van Geffen et al; ${ }^{33} 2009$

Jackevicius et al; ${ }^{34} 2008$ (a)

Jackevicius et al:;4 2008 (b)

Jackevicius et al; 342008 (c)

Jackevicius et al; ${ }^{34} 2008$ (d)

Karter et al; ${ }^{35} 2009$ (a)

Karter et al; 352009 (b)

Karter et al; ${ }^{35} 2009$ (c)

Kerner et al; 362017

Linnet et al; ${ }^{19} 2012$ (a)

Linnet et al; ${ }^{19} 2012$ (b)

Linnet et al; ${ }^{19} 2012$ (d)

O'Connor et al; ${ }^{37} 2014$

Raebel et al; ${ }^{25} 2012$ (a)

Raebel et al; ${ }^{25} 2012$ (b)

Raebel et al; ${ }^{25} 2012$ (c)

Shah et al; ${ }^{38} 2008$

Shah et al; ${ }^{39} 2009$

Shin et al; ${ }^{16} 2012$ (a)

Shin et al: ${ }^{16} 2012$ (b)

Shin et al; ${ }^{16} 2012$ (c)

Shin et al; ${ }^{16} 2012$ (d)

Tamblyn et al; ${ }^{40} 2014$ (a)

Tamblyn et al; ${ }^{40} 2014$ (b)

Tamblyn et al; ${ }^{40} 2014$ (c)

Thengilsdóttir et al; ;4 2015 (c)

Thengilsdóttir et al; ${ }^{14} 2015$ (d)

Trinacty et al; $; 2009$

Xing et al; ${ }^{42} 2011$

Overall $\left(I^{2}=99.7 \%, p=0.000\right)$
Primary nonadherence

ES $(95 \% \mathrm{Cl})$

$0.132(0.125-0.140)$

$0.075(0.073-0.077)$

$0.088(0.086-0.090)$

$0.115(0.113-0.117)$

$0.043(0.033-0.054)$

$0.432(0.399-0.464)$

$0.335(0.313-0.358)$

$0.710(0.688-0.731)$

$0.154(0.149-0.159)$

$0.184(0.174-0.195)$

$0.020(0.002-0.070)$

$0.064(0.040-0.095)$

$0.033(0.026-0.042)$

$0.064(0.045-0.089)$

$0.219(0.208-0.230)$

$0.195(0.190-0.199)$

$0.199(0.192-0.206)$

$0.214(0.206-0.221)$

$0.149(0.143-0.156)$

$0.042(0.031-0.057)$

$0.137(0.086-0.204)$

$0.064(0.058-0.071)$

$0.051(0.037-0.070)$

$0.326(0.191-0.485)$

$0.040(0.036-0.045)$

$0.032(0.029-0.035)$

$0.085(0.078-0.092)$

$0.222(0.028-0.600)$

$0.087(0.068-0.109)$

$0.086(0.077-0.095)$

$0.066(0.059-0.074)$

$0.133(0.119-0.147)$

$0.113(0.098-0.130)$

$0.070(0.063-0.078)$

$0.126(0.116-0.136)$

$0.150(0.130-0.172)$

$0.171(0.158-0.185)$

$0.126(0.120-0.131)$

$0.078(0.075-0.080)$

$0.223(0.218-0.229)$

$0.077(0.074-0.080)$

$0.291(0.263-0.321)$

$0.322(0.305-0.338)$

$0.336(0.319-0.354)$

$0.062(0.052-0.073)$

$0.080(0.074-0.086)$

$0.100(0.087-0.115)$

$0.131(0.104-0.162)$

$0.146(0.131-0.162)$

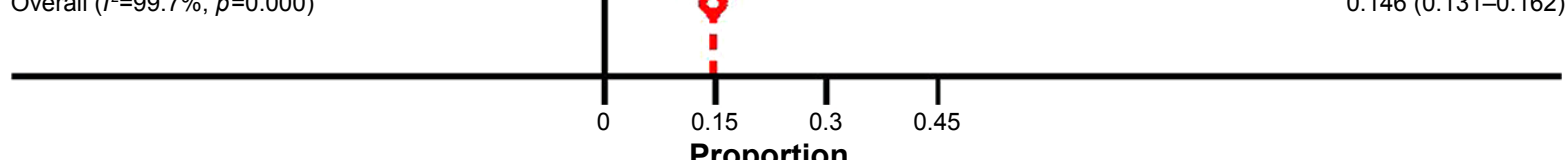

Figure 3 Forest plot for primary medication nonadherence.

Notes: (a) hypoglycemics; (b) anti-hypertensives; (c) lipid-lowering; (d) anti-depressants.

Abbreviation: ES, effect size.

on proper medication counseling, patient social support, and clinical follow-up to help reduce PMNA, especially where the indications for the prescribed medication aim to provide primary prevention.

\section{Strengths and limitations}

Our study has several strengths. There is a high level of congruence between our findings and those reported in the existing literature. However, our study provides a more 
clear and accurate picture of the PMNA impact because its reported effect estimates are not influenced by any single study. Additionally, the increased sample size obtained from pooling the effects of the included studies provides statistical strength.

Despite its strengths, our study has a few limitations. Given the nature of our study and its reliance on secondary data, we experienced some challenges in handling the residual (unmeasured) confounding effects that may be present within each study (eg, some of the included studies had identified their inability to assess the attitudes and beliefs of patients toward the prescribed medication, when these factors could clearly affect PMNA). Also, some of the values from the included studies might be either under- or overestimated because there is no way to independently verify whether patients either filled or did not fill their prescriptions from other sources or locations (ie, filled in different pharmacies or different states or provinces). Finally, some of the included studies were carried out with populations that could not be entirely generalizable and, therefore, should be interpreted with some level of caution.

\section{Disclosure}

The authors report no conflicts of interest in this work.

\section{References}

1. Sabaté E. Adherence to Long Term Therapies: Evidence for Action. World Health Organization; 2003. Available from: http://apps.who.int/iris/ bitstream/10665/42682/1/9241545992.pdf. Accessed November 13, 2017.

2. Lemstra M, Blackburn D, Crawley A, Fung R. Proportion and risk indicators of nonadherence to statin therapy: a meta-analysis. Can J Cardiol. 2012;28(5):574-580.

3. Chan B, Brossart B, Hudema N, et al. Improving the Quality of Heart Attack Care in Saskatchewan: Outcomes and Secondary Prevention. Saskatoon: Health Quality Council; 2004.

4. Iskedjian M, Addis A, Einarson T. Estimating the economic burden of hospitalization due to patient nonadherence in Canada. Value Health. 2002;5:470-471.

5. Chisholm-Burns M, Spivey C. The "cost" of medication nonadherence: consequences we cannot afford to accept. J Am Pharm Assoc. 2012; 52(6):823-826.

6. New England Healthcare Institute. Thinking Outside the Pillbox: A Systemwide Approach to Improving Patient Medication Adherence for Chronic Disease. 2009. Available from: https://www.nehi.net/writable/publication_files/file/pa_issue_brief_final.pdf. Accessed November 13, 2017.

7. May C, Montori V, Mair F. We need minimally disruptive medicine. BMJ. 2009;339(2):b2803.

8. Ting H, Shojania K, Montori V, Bradley E. Quality improvement: science and action. Circulation. 2009;119(14):1962-1974.

9. Zweifel P, Felder S, Meiers M. Ageing of population and health care expenditure: a red herring? Health Economics. 1999;8(6):485-496.

10. World Health Organization. Global Status Report on Noncommunicable Diseases. Geneva, Switzerland: World Health Organization; 2014. Available from: http://apps.who.int/iris/bitstream/10665/148114/1/97 89241564854_eng.pdf?ua=1. Accessed November 13, 2017.

11. World Health Organization. Depression. World Health Organization. 2017. Available from: http://www.who.int/mediacentre/factsheets/ fs369/en/
12. Fischer M, Stedman M, Lii J, et al. Primary medication non-adherence: analysis of 195,930 electronic prescriptions. J Gen Intern Med. 2010; 25(4):284-290.

13. Pottegård A, Christensen R, Houji A, et al. Primary non-adherence in general practice: a Danish register study. Eur J Clin Pharmacol. 2014; 70(6):757-763.

14. Thengilsdóttir G, Pottegård A, Linnet K, Halldórsson M, Almarsdóttir A, Gardarsdóttir H. Do patients initiate therapy? Primary non-adherence to statins and antidepressants in Iceland. Int J Clin Pract. 2015;69(5): 597-603.

15. Bauer A, Parker M, Schillinger D, et al. Associations between antidepressant adherence and shared decision-making, patient-provider trust, and communication among adults with diabetes: diabetes study of Northern California (DISTANCE). J Gen Intern Med. 2014;29:1139-1147.

16. Shin J, McCombs J, Sanchez R, Udall M, Deminski M, Cheetham C. Primary nonadherence to medications in an integrated healthcare setting. Am J Manag Care. 2012;18(8):426-434.

17. Fischer M, Choudhry N, Brill G, et al. Trouble getting started: predictors of primary medication nonadherence. Am J Med. 2011;124: 1081.e9-e22.

18. Lam W, Fresco P. Medication adherence measures: an overview. Biomed Res Int. 2015:217047.

19. Linnet K, Halldorsson M, Thengilsdottir G, Einarsson O, Jonsson K, Almarsdottir A. Primary non-adherence to prescribed medication in general practice: lack of influence of moderate increases in patient copayment. Fam Pract. 2012;30(1):69-75.

20. Higgins J, Green S. Cochrane Handbook for Systematic Reviews of Interventions Version 5.1.0 [updated March 2011]. The Cochrane Collaboration; 2011. Available from: http://www.handbook.cochrane.org. Accessed November 13, 2017.

21. Wells G, Shea B, O'Connell J, et al. The Newcastle-Ottawa Scale (NOS) for Assessing the Quality of Nonrandomised Studies in Meta-analysis. 2011. Available from: http://www.ohri.ca/programs/ clinical_epidemiology/oxford.asp. Accessed November 13, 2017.

22. Higgins J, Thompson S. Quantifying heterogeneity in a meta-analysis. Stat Med. 2002;21(11):1539-1558.

23. Tobias A. Assessing the influences of a single study in meta-analysis. Stata Tech Bull. 1999;47:15-17.

24. Begg C, Mazumdar M. Operating characteristics of a rank correlation test for publication bias. Biometrics. 1994;50(4):1088.

25. Raebel M, Ellis J, Carroll N, et al. Characteristics of patients with primary non-adherence to medications for hypertension, diabetes, and lipid disorders. J Gen Intern Med. 2012;27:57-64.

26. Aznar-Lou I, Fernández A, Gil-Girbau M, et al. Initial medication nonadherence: prevalence and predictive factors in a cohort of 1.6 million primary care patients. Br J Clin Pharmacol. 2017;83(6):1328-1340.

27. Casebeer L, Huber C, Bennett N, et al. Improving the physician-patient cardiovascular risk dialogue to improve statin adherence. BMC Fam Pract. 2009; 10:48.

28. Cheetham T, Niu F, Green K, et al. Primary nonadherence to statin medications in a managed care organization. J Manag Care Pharm. 2013; 19(5):367-373.

29. Derose S, Green K, Marrett E, et al. Automated outreach to increase primary adherence to cholesterol-lowering medications. JAMA Internal Medicine. 2013;173(1):38.

30. Ewen S, Meyer M, Cremers B, et al. Blood pressure reductions following catheter-based renal denervation are not related to improvements in adherence to antihypertensive drugs measured by urine/plasma toxicological analysis. Clin Res Cardiol. 2015;104(12):1097-1105.

31. Fischer M, Jones J, Wright E, et al. A randomized telephone intervention trial to reduce primary medication nonadherence. J Manag Care Spec Pharm. 2015;21(2):124-131.

32. Freccero C, Sundquist K, Sundquist J, Ji J. Primary adherence to antidepressant prescriptions in primary health care: a population-based study in Sweden. Scand J Prim Health Care. 2016;34(1):83-88. 
33. van Geffen E, Gardarsdottir H, van Hulten R, van Dijk L, Egberts A, Heerdink E. Initiation of antidepressant therapy: do patients follow the GP's prescription? Br J Gen Pract. 2009;59(559):81-87.

34. Jackevicius C, Li P, Tu J. Prevalence, predictors, and outcomes of primary nonadherence after acute myocardial infarction. Circulation. 2008;117(8):1028-1036.

35. Karter A, Parker M, Moffet H, Ahmed A, Schmittdiel J, Selby J. New prescription medication gaps: a comprehensive measure of adherence to new prescriptions. Health Serv Res. 2009;44(5 pt 1): 1640-1661.

36. Kerner D, Knezevich E. Use of communication tool within electronic medical record to improve primary nonadherence. $J$ Am Pharm Assoc. 2017;57(3):S270.e2-S273.e2.

37. O’Connor P, Schmittdiel J, Pathak R, Harris R, Newton K, Ohnsorg K. Randomized trial of telephone outreach to improve medication adherence and metabolic control in adults with diabetes. Diabetes Care. 2014;37:3317-3324

38. Shah N, Hirsch A, Zacker C, Taylor S, Wood G, Stewart W. Factors associated with first-fill adherence rates for diabetic medications: a cohort study. J Gen Intern Med. 2008;24(2):233-237.

39. Shah N, Hirsch A, Zacker C, et al. Predictors of first-fill adherence for patients with hypertension. Am J Hypertens. 2009;22(4):392-396.

40. Tamblyn R, Eguale T, Huang A, Winslade N, Doran P. The incidence and determinants of primary nonadherence with prescribed medication in primary care. Ann Intern Med. 2014;160(7):441.

41. Trinacty C, Adams A, Soumerai S, et al. Racial differences in long-term adherence to oral antidiabetic drug therapy: a longitudinal cohort study. BMC Health Serv Res. 2009;9:24.

42. Xing S, DiPaula B, Lee H, Cooke C. Failure to fill electronically prescribed antidepressant medications. Prim Care Companion CNS Disord. 2011:1-15.

43. Gadkari A, McHorney C. Medication nonfulfillment rates and reasons: narrative systematic review. Curr Med Res Opin. 2010;26(3):683-705.
44. Lemstra M, Alsabbagh M. Proportion and risk indicators of nonadherence to antihypertensive therapy: a meta-analysis. Patient Prefer Adherence. 2014;8:211-218.

45. Colhoun H, Betteridge D, Durrington P, et al; CARDS investigators. Primary prevention of cardiovascular disease with atorvastatin in type 2 diabetes in the Collaborative Atorvastatin Diabetes Study (CARDS): multicentre randomised placebo-controlled trial. Lancet. 2004;364(9435):685-696.

46. Kringos D, Boerma W, Van Der Zee J, Groenewegen P. Europe's strong primary care systems are linked to better population health but also to higher health spending. Health Aff (Millwood). 2013;32(4): 686-694.

47. Ridic G, Gleason S, Ridic O. Comparisons of health care systems in the United States, Germany and Canada. Mater Sociomed. 2012;24(2) $112-120$.

48. Lemstra M, Bird Y, Nwankwo C, Rogers M, Moraros J. Weight loss intervention adherence and factors promoting adherence: a metaanalysis. Patient Prefer Adherence. 2016;10:1547-1559.

49. DiMatteo M. Social support and patient adherence to medical treatment: a meta-analysis. Health Psychol. 2004;23(2):207-218.

50. Haynes RB, Yao X, Degani A, Kripalani S, Garg A, McDonald HP. Interventions for enhancing medication adherence. Cochrane Database Syst Rev. 2005;(4):CD000011.

51. Schedlbauer A, Davies P, Fayey T. Interventions to improve adherence to lipid lowering medication. Cochrane Database Syst Rev. 2010;(3): CD004371.

52. Nunes V, Neilson J, O'flynn N, et al. Clinical guidelines and evidence review for medicines adherence: involving patients in decisions about prescribed medicines and supporting adherence. London: National Collaborating Centre for Primary Care and Royal College of General Practitioners. 2009. Available from: http:/www.sefap.it/ servizi_lineeguida_200902/CG76FullGuidelineApp.pdf. Accessed November 13, 2017
Patient Preference and Adherence

\section{Publish your work in this journal}

Patient Preference and Adherence is an international, peer-reviewed, open access journal that focuses on the growing importance of patient preference and adherence throughout the therapeutic continuum. Patient satisfaction, acceptability, quality of life, compliance, persistence and their role in developing new therapeutic modalities and compounds to optimize

\section{Dovepress}

clinical outcomes for existing disease states are major areas of interest for the journal. This journal has been accepted for indexing on PubMed Central. The manuscript management system is completely online and includes a very quick and fair peer-review system, which is all easy to use. Visit http://www. dovepress.com/testimonials.php to read real quotes from published authors. 